\title{
MRAC with Time-varying Reference Model from Convex Combination
}

\author{
Kelley E. Hashemi and Nhan T. Nguyen
}

\begin{abstract}
An extension of model reference adaptive control is presented that accommodates use of a time-varying reference model. Specifically, the reference model is taken to be a time-varying convex combination of two linear, timeinvariant models. The design is intended to act as a way to smoothly transition between two different reference models without resorting to a scheduled switch. It also provides the ability to use an interpolated reference model when the plant is operating between design points. The time variation of the combination must satisfy some requirements to ensure stability but is otherwise user choice. Subject to these requirements, bounded tracking error behavior is demonstrated via Lyapunov stability analysis for the single-input, single-output, output feedback case. Tracking error convergence is asymptotic when time variation ceases. The proposed design is demonstrated in simulation of a numerical model.
\end{abstract}

\section{INTRODUCTION}

The design of the reference model is one of the primary degrees of freedom available to the user who is implementing any of the many schemes described as model reference adaptive control (MRAC). Few of these algorithms, however, attempt to exploit reference model design for additional gain, especially after the reference model is initially specified. Here we attempt to make use of this underutilized design choice by introducing a way to change the reference model online. The intent of the present work is to establish a framework accommodating time variation of the reference model within which more complex variations could be incorporated.

The framework considered in this paper replaces a traditional reference model with one that is a time-varying convex combination of two time-invariant reference models. The time variation is therefore isolated to a single parameter whose evolution is user choice but subject to some restrictions to ensure stability. Although simple, even this type of reference model manipulation has immediate applications. The most apparent use for such a design is as a smooth alternative to scheduling. Instead of requiring a discrete switch each time a reference model change is desired, a timevarying reference model can be used to continuously transition between the two with assured stability and without the disruptive switching transient. The smooth transition would be particular useful, for example, as the plant moves through a design space with multiple reference models available and selected based on operating condition.

The time-varying reference model structure also defines a continuum of reference models between any reference model

The authors are with the Intelligent Systems Division at NASA Ames Research Center, Moffett Field, CA, 94035 USA. kelley.e.hashemi@nasa.gov design points. At any given location in the design space an "interpolated" reference model can be in use rather than simply the reference model at the nearest design point. This feature could help improve off-nominal operation.

A small number of other studies have investigated mechanics which make the reference model time-varying. In [1], a time-varying reference model results from inclusion of updated parameter estimates in the reference model description. The time-varying reference model is then used to determine an optimal control solution at each time step. A class of formulations involving a reference model with error feedback term, often called closed-loop reference models, can also result in a time-varying reference model. The technique has been shown to improve management of tracking error and transient performance [2]-[4].

The notion of using convex combinations in the context of adaptive control is not entirely new. Specifically, in [5] parameter estimates arising from multiple models are combined in a convex fashion and used to improve upon the parameter estimates that would be in use otherwise. While this is not related to direct manipulation of the reference model in the same sense being discussed, it does result in some structures which also appear in our design. The interested reader may wish to compare treatment of the time-varying combination of parameters to that presented here.

The paper is organized as follows: Section II summarizes several established results that will be of use in developing the proposed controller. A general description of the problem structure is then provided in Section III. The error dynamics capturing the difference between the closed-loop plant and time-varying reference model are derived in Section IV and then used in Section V to establish tracking error behavior via Lyapunov stability analysis. Section VI presents a numerical example demonstrating the proposed design, and some comments on future work are made in Section VII.

\section{Mathematical Preliminaries}

A collection of known results useful in deriving the proposed control scheme is now provided. The first theorem establishes a linear matrix inequality (LMI) condition on a system perturbation that ensures maintained stability [6].

Theorem 1: Consider a stable system of the form $\dot{x}=A x$ such that there exists a $P=P^{T}>0$ and $Q=Q^{T}>0$ satisfying

$$
A^{T} P+P A=-Q .
$$

To assess whether the perturbed system $\dot{x}=(A+\Delta A) x$ 
remains stable, replace Eq. (2) with

$$
A^{T} P+P A=-Q-\Omega
$$

where $\Omega=\Omega^{T}>0$ and restate Eq. (4) with the perturbation

$$
\begin{aligned}
(A+\Delta A)^{T} P+P(A & +\Delta A)= \\
& -Q-\Omega+\left(\Delta A^{T} P+P \Delta A\right) .
\end{aligned}
$$

Thus, the perturbed system governed by $A+\Delta A$ will be stable so long as there exists an appropriate $\Omega$ such that

$$
\Delta A^{T} P+P \Delta A \leq-\Omega .
$$

The next result permits eigenvalue-based stability assessment for slowly time-varying systems [8].

Theorem 2: Consider the equation $\dot{x}(t)=A(t) x(t)$ with $A(t)$ continuously differentiable, $\|A(t)\| \leq \alpha \forall t$ for a selected $p$-norm, and each pointwise eigenvalue of $A(t)$ satisfying $\operatorname{Re}[\lambda(t)] \leq-\nu$ where $\alpha$ and $\nu$ are positive constants. If there exists a sufficiently small positive constant $\beta$ such that the condition

$$
\|\dot{A}(t)\| \leq \beta \quad \forall t
$$

is satisfied then the system is uniformly exponentially stable. Further discussion on the permissible size of $\beta$ can be found in texts such as Chapter 9.6 of [11], though in the context of this paper the parameter will be free for the user to assign such that Theorem 2 can always be satisfied.

Finally, a restricted version of the Meyer-KalmanYakubovich (MKY) lemma which provides time domain relationships for nonminimal representations of strictly positive real (SPR) systems is stated. The reader is referred to [9] or many other such texts for a formal characterization of SPR systems.

Theorem 3: Consider the stable system defined by the transfer function $G(s)=C^{T}(s I-A)^{-1} B$. If $G(s)$ is SPR then there exist matrices $Q=Q^{T}>0$ and $P=P^{T}>0$ such that

$$
\begin{gathered}
A^{T} P+P A=-Q \\
P B=C .
\end{gathered}
$$

\section{PRoblem Description}

Adhering to the notation of [9], consider the single-input single-output (SISO) plant given by transfer function $y=$ $G(s) u$ with

$$
G(s)=\rho^{*} \frac{Z_{p}(s)}{R_{p}(s)} .
$$

Here $u, y \in \mathbb{R}, Z_{p}(s)$ and $R_{p}(s)$ are monic polynomials, $R_{p}(s)$ is of degree $n$, and $\rho^{*}$ is a constant gain. The plant can be equivalently represented by the state space description $\left(A_{p}, B_{p}, C_{p}^{T}\right)$ with state $x_{p} \in \mathbb{R}^{n}$. Two SISO linear timeinvariant (LTI) reference submodels are selected as $y_{m_{1}}=$ $W_{m_{1}}(s) r$ and $y_{m_{2}}=W_{m_{2}}(s) r$ with

$$
W_{m_{1}}(s)=\frac{Z_{m_{1}}(s)}{R_{m_{1}}(s)}
$$

where $Z_{m_{1}}(s)$ and $R_{m_{1}}(s)$ are monic polynomials. $W_{m_{2}}(s)$ is specified similarly. A single linear time-varying (LTV) reference model $y_{m}=W_{m}(s, t) r$ is constructed from the two LTI submodels by forming the convex combination

$$
W_{m}(s, t)=c(t) W_{m_{1}}(s)+(1-c(t)) W_{m_{2}}(s)
$$

where $c(t) \in[0,1]$ and $r, y_{m} \in \mathbb{R}$. The reference input $r$ is taken to be uniformly bounded and piecewise continuous. Note that transitioning $c(t)$ from 0 to 1 changes the reference model from $W_{m_{2}}$ to $W_{m_{1}}$.

For the purposes of this paper, the following requirements are placed on the plant and reference model:

- $R_{m}(s), Z_{m}(s)$, and $Z_{p}(s)$ are monic Hurwitz polynomials. $R_{m}(s)$ is of degree $p_{m} \leq n$.

- Relative degree $=1$ for both $G(s)$ and $W_{m}(s, t)$.

- The sign of $\rho^{*}$ is known.

- $W_{m_{1}}(s)$ and $W_{m_{2}}(s)$ are SPR.

While some of these assumptions can be relaxed, such features are not discussed here to facilitate a simple discussion. Function arguments are also dropped were possible, but may be retained to emphasize time dependency caused by $c(t)$.

The goal is to design a model reference adaptive control law $u$ and smooth transition function $c(t)$ such that the output of the plant matches the output of the time-varying reference model as close as possible for any appropriate $r$.

\section{ERROR DYNAMICS}

This section builds the elements necessary to state a useful form of the error dynamics relating the reference model and closed-loop plant for the chosen controller structure. First a feasible matching condition is found showing that there are ideal time-varying parameters that will force the plant and reference model to match. It is also shown that the time-varying parameters are a convex combination of the submodels' fixed ideal parameters. Next, a composite system representation is built for the plant using this parameter relationship and is shown to be stable. Finally, the reference model dynamics are similarly stated and error dynamics are formed.

\section{A. Control Structure}

The controller is taken to be of an established form utilized by relative degree $=1$ output feedback MRAC designs [9] and is given by

$$
u=\theta^{T} \omega
$$

where $\theta=\left[\begin{array}{lll}\theta_{1}^{T} & \theta_{2}^{T} & \frac{1}{\rho}\end{array}\right]^{T} \in \mathbb{R}^{2 n+1}$ contains the parameters to be updated online and $\omega=\left[\begin{array}{lll}\omega_{1}^{T} & \omega_{2}^{T} & r\end{array}\right]^{T} \in \mathbb{R}^{2 n+1}$ is known as the regressor. The regressor quantities are calculated according to

$$
\begin{array}{ll}
\dot{\omega}_{1}=F \omega_{1}+g u, & \omega_{1}(0)=0 \\
\dot{\omega}_{2}=F \omega_{2}+g y, & \omega_{2}(0)=0
\end{array}
$$

where the matrices are defined according to the multi-output transfer function

$$
(s I-F)^{-1} g=\frac{\alpha(s)}{\Lambda(s)}
$$


with $\alpha(s)=\left[\begin{array}{llll}s^{n-1} & s^{n-2} \ldots s & 1\end{array}\right]^{T}$ and $\Lambda(s)$ a monic, stable polynomial of degree $n$ specified subsequently. The closedloop plant formed using this controller is denoted by $G_{\mathrm{cl}}(s)$.

\section{B. Matching Condition}

First consider the individual matching conditions implied by forcing the plant to match each submodel in turn. Let the closed-loop plant match the first LTI submodel model given in an expanded form as

$$
W_{m_{1}}=\frac{Z_{m_{1}} R_{m_{2}}}{R_{m_{1}} R_{m_{2}}}
$$

when using ideal parameters denoted by the vector

$$
\theta_{m_{1}}^{*}=\left[\begin{array}{ccc}
\theta_{1}^{* T} & \theta_{2}^{* T} & 1 \\
m_{1} & m_{1} & \rho^{*}
\end{array}\right]^{T} .
$$

such that $y_{m_{1}}=W_{m_{1}}(s) r=G(s) \theta_{m_{1}}^{* T} \omega$. Note that in this paper $\rho^{*}$ is taken to have the same value for both submodels. Select the polynomial

$$
\Lambda_{1}=\Lambda_{0} Z_{m_{1}} R_{m_{2}}
$$

where $\Lambda_{0}$ is a monic, stable polynomial of appropriate dimension. It can be shown that for $G_{\mathrm{cl}}(s)=W_{m_{1}}(s)$ to hold, the matching condition

$$
\left(\begin{array}{c}
\Lambda_{1}-\theta_{1}^{* T} \alpha \\
m_{1}
\end{array}\right) R_{p}-\rho^{*} Z_{p} \theta_{m_{1}}^{* T} \alpha=Z_{p} \Lambda_{0} R_{m_{1}} R_{m_{2}}
$$

must be satisfied in the case of ideal parameter knowledge (see [9] Chapter 6.3). Satisfaction of this condition is feasible so long as $\Lambda_{1}$ and $Z_{p}(s)$ are Hurwitz so that pole/zero cancellations occurring because of matching happening in the left half plane (LHP). Repeating the same analysis for the second LTI reference model with

$$
W_{m_{2}}=\frac{Z_{m_{2}} R_{m_{1}}}{R_{m_{2}} R_{m_{1}}}
$$

and

$$
\Lambda_{2}=\Lambda_{0} Z_{m_{2}} R_{m_{1}}
$$

leads to the matching condition

$$
\left(\begin{array}{c}
\Lambda_{2}-\theta_{1}^{* T} \alpha \\
m_{2}
\end{array}\right) R_{p}-\rho^{*} Z_{p} \theta_{m_{2}}^{* T} \alpha=Z_{p} \Lambda_{0} R_{m_{1}} R_{m_{2}}
$$

that ensures $G_{\mathrm{cl}}(s)=W_{m_{2}}(s)$ is satisfied.

Next, if $G_{\mathrm{cl}}(s)=W_{m}(s, t)$ is to hold, select $\Lambda$ to be a varying convex combination of $\Lambda_{1}$ and $\Lambda_{2}$

$$
\Lambda(s, t)=\Lambda_{0}\left[c(t) Z_{m_{1}} R_{m_{2}}+(1-c(t)) Z_{m_{2}} R_{m_{1}}\right]
$$

with $\Lambda_{0}$ reselected as necessary. The same kind of analysis can be used to show that the matching condition

$$
\begin{aligned}
& \left(\Lambda(s, t)-\theta_{1}^{* T}(t) \alpha\right) R_{p}-\rho^{*} Z_{p} \theta_{2}^{* T}(t) \alpha= \\
& Z_{p} \Lambda_{0} R_{m_{1}} R_{m_{2}}
\end{aligned}
$$

is required where the ideal parameters are now time-varying to account for inclusion of $c(t)$. Note that the relationship in Eq. (16) indicates that $F$ will also vary, which will be discussed in more detail later. Further, it can be shown that $\theta^{*}(t)$ is formed using the ideal parameters associated with the individual submodels as shown in the following theorem.

Theorem 4: Consider the plant and reference model described in Section III with controller given in Eq. (14). When $\Lambda(s, t)$ given by Eq. (24) remains Hurwitz for all $c(t) \in[0,1]$, a varying convex combination of the ideal parameter vectors $\theta_{m_{1}}^{*}$ and $\theta_{m_{2}}^{*}$ implied by Eqs. (20) and (23) can be used to form $\theta^{*}(t)$ according to

$$
\theta^{*}(t)=c(t) \theta_{m_{1}}^{*}+(1-c(t)) \theta_{m_{2}}^{*}
$$

which satisfies the matching condition in Eq. (25).

Proof: First note that the matching condition in Eq. (25) will only be feasible if $\Lambda$ remains Hurwitz as $c(t)$ varies. Since this value is restricted to be between 0 and 1, closed-form tests exist that can be used to check that each possible polynomial that results is stable [7], and the reference submodels can be redesigned to meet this requirement if not.

To demonstrate that a convex combination structure for $\theta^{*}(t)$ is sufficient for matching, start from the left side of the matching condition in Eq. (25), denoted by $L$ for brevity, and substitute the choice for $\Lambda$ given in Eq. (24) to obtain the polynomial

$$
\begin{gathered}
L=\left(\Lambda_{0}\left[c(t) Z_{m_{1}} R_{m_{2}}+(1-c(t)) Z_{m_{2}} R_{m_{1}}\right]\right. \\
\left.-\theta_{1}^{* T} \alpha\right) R_{p}-\rho^{*} Z_{p} \theta_{2}^{* T} \alpha .
\end{gathered}
$$

Next utilize the proposed convex combination expression for $\theta^{*}(t)$ given in Eq. (26) and regroup to reach

$$
\begin{gathered}
L=c(t)\left[\left(\Lambda_{0} Z_{m_{1}} R_{m_{2}}-\theta_{1}^{* T} \alpha\right) R_{p}-\rho^{*} Z_{p} \theta_{2}^{* T} \alpha\right] \\
+(1-c(t))\left[\left(\Lambda_{0} Z_{m_{2}} R_{m_{1}}-\theta_{1}^{* T} \alpha\right) R_{p}-\rho^{*} Z_{p} \theta_{2}^{* T} \alpha\right] .
\end{gathered}
$$

Substituting the equalities provided by the submodel matching conditions in Eqs. (20) and (23) reduces the previous expression to

$$
L=c(t)\left(Z_{p} \Lambda_{0} R_{m_{1}} R_{m_{2}}\right)+(1-c(t))\left(Z_{p} \Lambda_{0} R_{m_{1}} R_{m_{2}}\right)
$$

or $Z_{p} \Lambda_{0} R_{m_{1}} R_{m_{2}}$ which is equivalent to the right side of the proposed matching condition stated in Eq. (25).

\section{Composite System Construction}

To facilitate formulation of the error dynamics, create a closed-loop state space representation of the plant described in Eq. (11)

$$
\begin{aligned}
\dot{Y}_{c} & =A_{0}(t) Y_{c}+B_{c} u \\
y & =C_{c}^{T} Y_{c}
\end{aligned}
$$

where the state vector is $Y_{c}=\left[\begin{array}{lll}x_{p}^{T} & \omega_{1}^{T} & \omega_{2}^{T}\end{array}\right]^{T}$ and

$$
\begin{aligned}
A_{0}(t) & =\left[\begin{array}{ccc}
A_{p} & 0 & 0 \\
0 & F(t) & 0 \\
g C_{p}^{T} & 0 & F(t)
\end{array}\right] \\
B_{c}^{T} & =\left[\begin{array}{lll}
B_{p}^{T} & g^{T} & 0
\end{array}\right]
\end{aligned}
$$




$$
C_{c}^{T}=\left[\begin{array}{lll}
C_{p}^{T} & 0 & 0
\end{array}\right] .
$$

Adding and subtracting $B_{c} \theta^{* T}(t) \omega$ gives

$$
\dot{Y}_{c}=A_{c}(t) Y_{c}+B_{c} \frac{1}{\rho^{*}} r+B_{c}\left(u-\theta^{* T} \omega\right) .
$$

Note that $A_{c}(t)$ can be decomposed as

$$
\begin{aligned}
A_{c}(t) & =A_{0}(t)+B_{0}(t) \\
& =\left[\begin{array}{ccc}
A_{p} & 0 & 0 \\
0 & F(t) & 0 \\
g C_{p}^{T} & 0 & F(t)
\end{array}\right]+\left[\begin{array}{ccc}
0 & B_{p} \theta_{1}^{* T}(t) & B_{p} \theta_{2}^{* T}(t) \\
0 & g \theta_{1}^{* T}(t) & g \theta_{2}^{* T}(t) \\
0 & 0 & 0
\end{array}\right]
\end{aligned}
$$

Additionally, the following theorem establishes that $A_{c}(t)$ can be expressed in terms of the constant $A_{c}$ matrices associated with each of the submodels-denoted as $A_{m_{1}}$ and $A c$-and will remain stable for some $c(t)$.

Theorem 5: Consider the matrix $A_{c}(t)$ and its decomposition given in Eq. (35) along with the definitions for $F(t)$ in Eq. (16), $\Lambda(s, t)$ in Eq. (24), and $\theta^{*}(t)$ in Eq. (26). $A_{c}(t)$ is equivalent to the convex combination

$$
A_{c}(t)=c(t) A_{m_{1}}^{c}+(1-c(t)) A_{m_{2}}^{c}
$$

where $A_{m_{1}}$ and $A_{m_{2}}$ are the constant matrices obtained from the $A_{c}(t)$ expression when $c=1$ and $c=0$, respectively.

Further, $A_{c}(t)$ is uniformly exponentially stable when $c(t)$ is bounded and continuously differentiable, $\dot{c}(t)$ is bounded by a sufficiently small number, and the matching conditions discussed in Section IV-B are feasible.

Proof: From Eq. (16), note that the elements of $F(t)$ are defined by the coefficients of $\Lambda(s, t)$. Since $\Lambda(s, t)$ is a convex combination of the $\Lambda_{1}(s)$ and $\Lambda_{2}(s)$ polynomials associated with each submodel, it is permissible to express $F(t)$ as a convex combination of the $F$ matrices for each submodel as well according to

$$
F(t)=c(t) F_{m_{1}}+(1-c(t)) F_{m_{2}} .
$$

Here $F_{m_{1}}$ is defined by $\Lambda_{1}(s)$ in Eq. (19) and $F_{m_{2}}$ by Eq. (22). Then, take $A_{0}$ to be the matrix in Eq. (31) containing $F_{m_{1}}$ instead of $F(t)$ and similar for $A_{m_{2}}$ such that

$$
A_{0}(t)=c(t) \underset{m_{1}}{A_{0}}+(1-c(t)) A_{m_{2}} .
$$

The convex combination expression in Eq. (26) permits

$$
B_{0}(t)=c(t) B_{m_{1}}^{0}+(1-c(t)) B_{m_{2}} .
$$

Therefore,

$$
\begin{aligned}
& A_{c}(t)=c(t)\left(\begin{array}{c}
A_{0} \\
m_{1}
\end{array}+B_{m_{1}}\right)+(1-c(t))\left(\begin{array}{c}
A_{m_{2}} \\
m_{m_{2}}
\end{array}\right) \\
& =c(t) A_{m_{1}}^{c}+(1-c(t)) A_{m_{2}} .
\end{aligned}
$$

To establish stability of $A_{c}(t)$, note that

$$
G_{\mathrm{cl}}(s, t)=C_{c}^{T}\left(s I-A_{c}(t)\right)^{-1} B_{c} \frac{1}{\rho^{*}}=W_{m}(s, t)
$$

when the matching condition in Eq. (25) is satisfied. Recall that all necessary pole/zero cancellation will occur in the LHP. Combining this with the design of $W_{m}(s, t)$ means that all (time-varying) eigenvalues of $A_{c}(t)$ are also in the LHP. Then, so long as $c(t)$ is such that the remaining requirements of Theorem 2 are satisfied, $A_{c}(t)$ provides uniform exponential stability.

\section{Composite System Error}

The proof of Theorem 5 highlights the fact that $W_{m}(s, t)=C_{c}^{T}\left(s I-A_{c}(t)\right)^{-1} B_{c} \frac{1}{\rho^{*}}$ under the matching condition. Correspondingly, a nonminimal representation for reference model dynamics is given by

$$
\begin{aligned}
\dot{Y}_{m} & =A_{c}(t) Y_{m}+B_{c} \frac{1}{\rho^{*}} r \\
y_{m} & =C_{c}^{T} Y_{m} .
\end{aligned}
$$

Defining $e=Y_{c}-Y_{m}, e_{1}=y-y_{m}$, and $\bar{B}_{c}=B_{c} \frac{1}{\rho^{*}}$ while selecting $u=\theta^{T}(t) \omega$ yields the error dynamics

$$
\begin{aligned}
\dot{e} & =A_{c}(t) e+\bar{B}_{c} \rho^{*} \tilde{\theta}^{T}(t) \omega \\
e_{1} & =C_{c}^{T} e
\end{aligned}
$$

where $\tilde{\theta}(t)=\theta(t)-\theta^{*}(t)$.

\section{Stability Analysis}

The control system design and requirements necessary to demonstrate tracking error convergence are presented in the following theorem.

Theorem 6: For the plant and time-varying reference model described in Section III, the controller in Eq. (14), and the parameter update law

$$
\dot{\theta}=-\operatorname{sign}\left(\rho^{*}\right) \Gamma e_{1} \omega
$$

ensure that the tracking error $e_{1}(t)=y(t)-y_{m}(t)$ converges to and subsequently remains in a residual set so long as

1) $c(t) \in[0,1]$, is continuously differentiable if $\dot{c}(t) \neq 0$, and satisfies $|\dot{c}(t)|<\beta$ for all $t \geq 0$ and some sufficiently small, positive constant $\beta$.

2) $\Delta A=A_{m_{1}}-A_{m_{2}}$ must satisfy the bounded relationship of Theorem ${ }^{m_{2}}$ with the MKY matrix arising from $\left(A_{m_{2}}, B_{c}, C_{c}^{T}\right)$.

3) $\tilde{\theta}$ remains bounded.

4) $\Lambda(s, t)$ remains Hurwitz for all $c(t) \in[0,1]$.

Further, should time variation cease and $\dot{c}(t)=0$, tracking error will converge asymptotically.

Proof: First note that Requirement 4 and the $c(t)$ bounded and continuously differentiable portion of Requirement 1 are necessary only to ensure Theorems 4 and 5 are satisfied. Then select the Lyapunov function

$$
V(e, \tilde{\theta})=\frac{1}{2} e^{T} P e+\frac{\left|\rho^{*}\right|}{2} \tilde{\theta}^{T} \Gamma^{-1} \tilde{\theta}
$$

with $\Gamma=\Gamma^{T}>0$ and $P=P^{T}>0$ a constant matrix defined subsequently.

Taking the derivative leads to

$$
\begin{aligned}
\dot{V}=\frac{1}{2} e^{T}\left(A_{c}(t)^{T} P\right. & \left.+P A_{c}(t)\right) e \\
& +e^{T} P \bar{B}_{c} \rho^{*} \tilde{\theta}^{T} \omega+\left|\rho^{*}\right| \tilde{\theta}^{T} \Gamma^{-1} \dot{\tilde{\theta}} .
\end{aligned}
$$

Since $A_{c}(t)$ can be expressed as

$$
\begin{aligned}
A_{c}(t) & =\underset{m_{2}}{A_{2}}+c(t)\left(A_{m_{1}}^{c}-A_{m_{2}}^{c}\right) \\
& =\underset{m_{2}}{c}+c(t) \Delta A,
\end{aligned}
$$


take $P$ to be a symmetric, positive definite matrix satisfying the MKY lemma for the nonminimal representation of the SPR submodel $\left(A_{m_{2}}^{c}, \bar{B}_{c}, C_{c}^{T}\right)$

$$
\begin{gathered}
A_{m_{2}}^{A_{c}} P+P A_{m_{2}}=-Q \\
P \bar{B}_{c}=C_{c}
\end{gathered}
$$

as well as the additional relationship

$$
\Delta A^{T} P+P \Delta A \leq-\Omega .
$$

Then by using Theorem 1 it is possible to express the bound

$$
\begin{aligned}
A_{c}^{T} & (t) P+P A_{c}(t) \\
& =\left(A_{m_{2}}+c(t) \Delta A\right)^{T} P+P\left(A_{m_{2}}+c(t) \Delta A\right) \\
& =-Q+c(t)\left(\Delta A^{T} P+P \Delta A\right) \\
& \leq-Q-c(t) \Omega
\end{aligned}
$$

The derivative can then be bounded by

$$
\dot{V} \leq-\frac{1}{2} e^{T} Q e+e_{1} \rho^{*} \tilde{\theta}^{T} \omega+\left|\rho^{*}\right| \tilde{\theta}^{T} \Gamma^{-1} \dot{\tilde{\theta}}
$$

where the scalar $e_{1}=e^{T} P \bar{B}_{c}$ due to Eq. (49).

It is important to note that $\dot{\tilde{\theta}}=\dot{\theta}-\dot{\theta}^{*}$ here due to the dependence of $\theta^{*}$ on $c(t)$, which is a departure from typical MRAC stability analysis. Recalling Eq. (26) yields

$$
\dot{\tilde{\theta}}=\dot{\theta}-\dot{\theta^{*}}=\dot{\theta}-\dot{c}\left(\theta_{m_{1}}^{*}-\theta_{m_{2}}^{*}\right)
$$

and as a result an additional term is generated in $\dot{V}$ such that

$$
\begin{aligned}
\dot{V} \leq-\frac{1}{2} e^{T} Q e+e_{1} \rho^{*} \tilde{\theta}^{T} \omega+\left|\rho^{*}\right| \tilde{\theta}^{T} \Gamma^{-1} \dot{\theta} & \\
& -\left|\rho^{*}\right| \tilde{\theta}^{T} \Gamma^{-1} \dot{c}\left(\theta_{m_{1}}^{*}-\theta_{m_{2}}^{*}\right) .
\end{aligned}
$$

Selecting the update law $\dot{\theta}=-\operatorname{sign}\left(\rho^{*}\right) \Gamma e_{1} \omega$ leaves the expression

$$
\dot{V} \leq-\frac{1}{2} e^{T} Q e-\dot{c}\left|\rho^{*}\right| \tilde{\theta}^{T} \Gamma^{-1}\left(\theta_{m_{1}}^{*}-\theta_{m_{2}}^{*}\right) .
$$

From here it is clear that if $\dot{c}=0$ then $\dot{V} \leq-\frac{1}{2} e^{T} Q e \leq$ 0 and asymptotic convergence of the tracking error can be demonstrated with a signal chasing argument and Barbalat's Lemma [11].

Now consider the $\dot{c}>0$ and $\dot{c}<0$ cases. To proceed, define $V_{0}=\frac{1}{2} e^{T} P e$ and $V_{1}=\frac{\left|\rho^{*}\right|}{2} \tilde{\theta} \Gamma^{-1} \tilde{\theta}$ such that $V=$ $V_{0}+V_{1}$ and note that the following relationships hold:

$$
\begin{aligned}
& -\frac{1}{2} e^{T} Q e \leq-\frac{\lambda_{\min }(Q)}{\lambda_{\max }(P)} V_{0} \\
& -\dot{c}\left|\rho^{*}\right| \tilde{\theta}^{T} \Gamma^{-1} \tilde{\theta}=-2 \dot{c} V_{1} .
\end{aligned}
$$

Adding and subtracting the quantity $\dot{c}\left|\rho^{*}\right| \tilde{\theta}^{T} \Gamma^{-1} \tilde{\theta}$ from Eq. (55) and utilizing the previous two expressions allow the derivative to be bounded as

$$
\begin{aligned}
\dot{V} \leq- & {\left[\frac{\lambda_{\min }(Q)}{\lambda_{\max }(P)} V_{0}+2|\dot{c}| V_{1}\right] } \\
& +|\dot{c}|\left|\rho^{*}\right| \tilde{\theta}^{T} \Gamma^{-1}\left[\tilde{\theta}-\operatorname{sign}(\dot{c})\left(\theta_{m_{1}}^{*}-\theta_{m_{2}}^{*}\right)\right] .
\end{aligned}
$$

Here $\dot{c}=\operatorname{sign}(\dot{c})|\dot{c}|$ has been used to facilitate addressing both directions of growth in a single expression. If the boundedness requirement $|\dot{c}| \leq \beta$ holds then

$$
\begin{aligned}
\dot{V} \leq & -\left[\frac{\lambda_{\min }(Q)}{\lambda_{\max }(P)} V_{0}+2|\dot{c}| V_{1}\right] \\
& +\beta\left|\rho^{*}\right|\left[\tilde{\theta}^{T} \Gamma^{-1} \tilde{\theta}+\left|\tilde{\theta}^{T} \Gamma^{-1}\left(\theta_{m_{1}}^{*}-\theta_{m_{2}}^{*}\right)\right|\right] .
\end{aligned}
$$

Define

$$
\mu=\min \left[\frac{\lambda_{\min }(Q)}{\lambda_{\max }(P)}, 2|\dot{c}|\right]
$$

as well as

$$
\delta \geq \beta\left|\rho^{*}\right|\left[\tilde{\theta}^{T} \Gamma^{-1} \tilde{\theta}+\left|\tilde{\theta}^{T} \Gamma^{-1}\left(\theta_{m_{1}}^{*}-\theta_{m_{2}}^{*}\right)\right|\right]
$$

where the quantity $0 \leq \delta<\infty$ so long as $\tilde{\theta}$ remains bounded, such as with projection. Finally, the derivative can be written as

$$
\dot{V} \leq-\mu\left(V_{0}+V_{1}\right)+\delta=-\mu V+\delta .
$$

$\dot{V}<0$ whenever $V>\frac{\delta}{\mu}$ thus defining a residual set where $V$ remains once it has entered. Similarly, $e$ and therefore $e_{1}$ can be shown to remain in a residual set due to their constant relationship.

Remark 1: Requirement 2 of Theorem 6 is conservative. Significant relaxation of this point has been seen to still yield tracking error convergence in simulation and is a source of ongoing investigation.

\section{EXAMPLE}

While the proposed convex combination MRAC scheme can of course stabilize systems meeting the outlined requirements, instead consider the more complex example of stabilizing a system with time varying linear perturbations. Such a situation might arise when designing a controller for an aircraft as it changes flight conditions. In this example the plant dynamics begin at

$$
y=\frac{-2(s+5)}{(s-1)^{2}} u .
$$

The plant dynamics gradually transition to

$$
y=\frac{-2(s+5)}{(s-4)^{2}} u
$$

over the course of the simulation. Both sets of plant dynamics meet the stated structural requirements. Two reference models are given by

$$
y_{m_{1}}=\frac{1}{(s+3)} r \quad y_{m_{2}}=\frac{1}{(s+1)} r
$$

such that their convex combination is given by

$$
\begin{aligned}
y_{m} & =c(t) y_{m_{1}}+(1-c(t)) y_{m_{2}} \\
& =\frac{s+(3-2 c(t))}{(s+3)(s+1)} r .
\end{aligned}
$$

Note that $\operatorname{sign}\left(\rho^{*}\right)=-1$. In this case select

$$
\Lambda(s, t)=\Lambda_{0}(s)[s+(3-2 c(t))]
$$


with $\Lambda_{0}(s)=(s+0.4)$ to maintain the degree $n$ requirement. Also select $r=15$ and $\Gamma=I$.

The control design is implemented assuming that the timevarying plant is unknown and only the output is available for feedback. Three reference models are considered: 1) The first reference model remains fixed. 2) The reference model discretely switches from the first to the second at $t=1.5$ s. 3) The proposed convex combination reference model is used with transition governed by $c(t)=e^{-0.4 t}$. The output tracking performance in each of the three cases is shown in Fig. 1, and the corresponding norm of the output tracking error is given in Fig. 2. From the simulation results it is clear
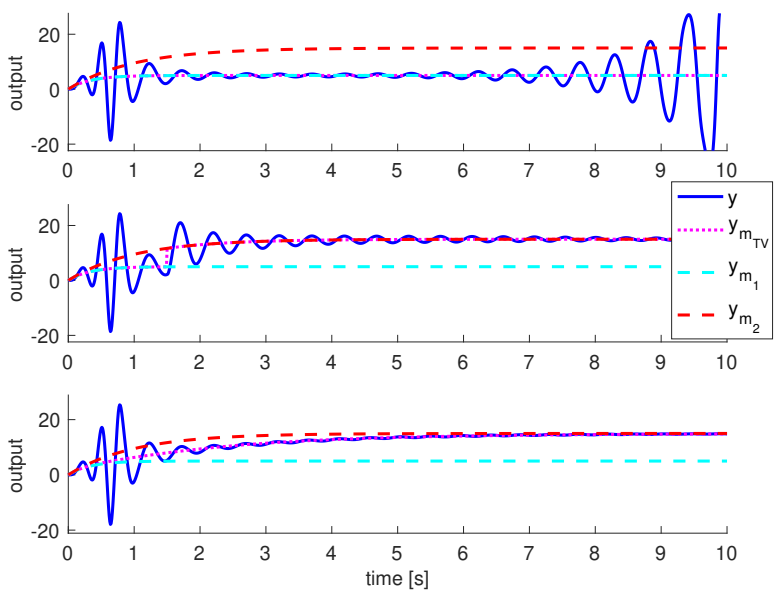

Fig. 1. Comparison of tracking performance with no reference model switch (top), discrete switch (middle), switch governed by $c(t)$ (bottom)

that for the example presented maintaining a fixed reference model did not provide a stabilizing control design. Use of both the switched and the convex combination reference models did result in stabilizing controllers, however the transient performance of the convex combination is clearly an improvement over the discrete switch.

\section{CONCLUSIONS}

A version of model reference adaptive control utilizing a time-varying reference model constructed from a variable convex combination of time-invariant models was proposed. Requirements on the time variation of the convex combination were developed in the course of demonstrating bounded tracking error behavior. A numerical simulation was presented to demonstrate the proposed design. In the future, less restrictive design requirements on both the invariant reference model components as well as their varying
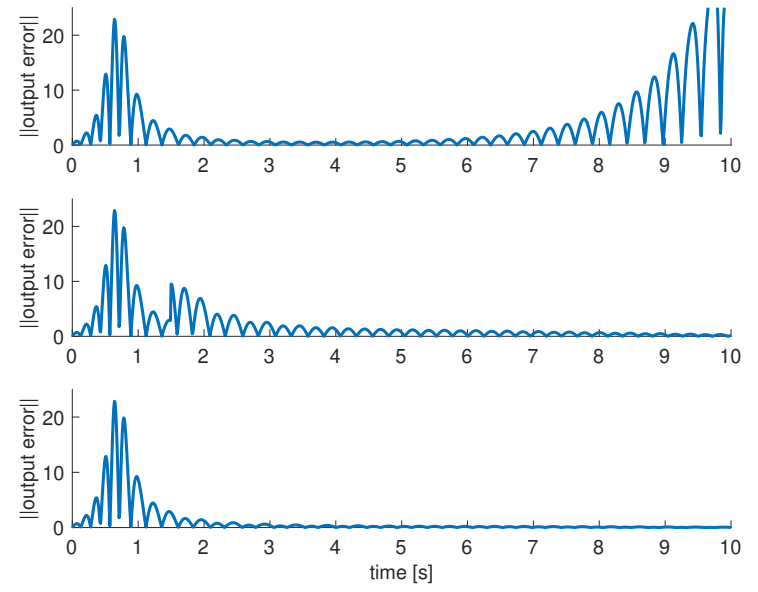

Fig. 2. Tracking error with no reference model switch (top), discrete switch (middle), switch governed by $c(t)$ (bottom)

combination are sought. Immediate application to existing systems that rely on gain-scheduled behavior is also under development. Additionally, extension to convex combinations of more than two reference model components will be used to span a multi-dimensional design space.

\section{REFERENCES}

[1] N.T. Nguyen, K.E. Hashemi, T. Yucelen, and E. Arabi, "Performance optimizing multi-objective adaptive control with time-varying model reference modification," AIAA SciTech, Grapevine, TX, Jan 2017.

[2] V. Stepanyan and K. Krishnakumar, "Adaptive control with reference model modification," AIAA Journal of Guidance, Control, and Dynamics, vol. 35, no. 4, pp. 1370-1374, 2012.

[3] E. Lavretsky, "Adaptive output feedback design using asymptotic properties of LQG/LTR controllers," IEEE Trans. on Automatic Control, vol. 57, no. 6, June 2012, pp. 1587-1591.

[4] T.E. Gibson, A.M. Annaswamy, and E. Lavretsky, "On adaptive control with closed-loop reference models: transients, oscillations, and peaking," IEEE Access, vol. 1, pp. 703-717, 2013.

[5] Z. Han and K.S. Narendra, "New concepts in adaptive control using multiple models," IEEE Trans. on Automatic Control, vol. 57, no. 1, pp. 78-89, 2012.

[6] W.M. Haddad and D.S. Bernstein, "Parameter-dependent Lyapunov functions, constant real parameter uncertainty, and the Popov criterion in robust analysis and synthesis part I,' IEEE CDC, Brighton, England, Dec 1991.

[7] S. Bialas, "A necessary and sufficient condition for stability of the convex combination of polynomials," Control and Cybernetics, vol. 33, no. 4, pp. 589-597, 2004.

[8] W.J. Rugh, Linear Systems Theory, Upper Saddle River, NJ: PrenticeHall, 1996.

[9] P.A. Ioannou and J. Sun, Robust Adaptive Control, Mineola, NY: Dover, 2012.

[10] R. Bellman, Stability Theory of Differential Equations New York: McGraw-Hill, 1953.

[11] H.K. Khalil, Nonlinear Systems, Upper Saddle River, NJ: PrenticeHall, 2002. 